\title{
Pesticide induced complications among pistachio farmers: In the rural area of Rafsanjan, Iran (2010)
}

\author{
Tavakolian Ferdosieyeh V, MS ${ }^{1}$, Karimifared M, MD ${ }^{2}$, Esmaeili A, $\mathrm{PhD}^{3}$, , Hasanshahi GH, $\mathrm{PhD}^{4}$, \\ Vazirinejad $\mathrm{R}, \mathrm{PhD}^{5 *}$ \\ 1- Medical Students, Medical School, Rafsanjan University of Medical Sciences, Rafsanjan, Iran. 2- Physician, Medical School, \\ Rafsanjan University of Medical Sciences, Rafsanjan, Iran. 3- Associate Prof., of Social Medicine, University of Medical \\ Sciences Rafsanjan, Iran. 4- Associate Prof., of Hematology, Molecular Medicine Research center, University of Medical \\ Sciences Rafsanjan, Iran. 5- Associate Prof., of Social Medicine, University of Medical Sciences Rafsanjan, Iran.
}

\begin{abstract}
Received: July 2012, Accepted: October 2012

Background: Pesticides have important effects on human health. Due to the wide range of pesticide application and also involvement of more than $60 \%$ of people living in this study area in the agriculture, it is essential to assess the side effects of these biohazards. Therefore, we designed this study to assess the impact of using pesticides on the health status of pistachio farmers, in rural area of Rafsanjan, Iran.

Material and Methods: In this descriptive study we have analysed the side effects of pesticides on farmers who used these substances in pistachio farms. A sample of 115 male farmers in the rural area of Rafsanjan County was randomly recruited. A questionnaire was designed to ask about age, marrital

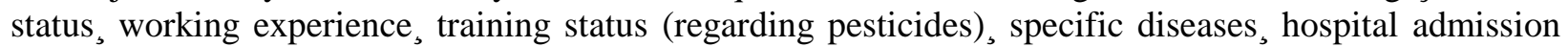
the history of acute poisoning and other toxic signs of respondents. Data were analysed using SPSS and Excel. Parametric and non-parametric tests were used to compare groups where appropriate.

Results: One hundred and four cases were married of which $1.7 \%$ were infertile. Among respondents' children (102), we found 6 cases of brain disease that 2 of them suffered from menangocell. About $45 \%$ of respondents did not change their diet during application of pesticides. Only $14.7 \%$ of respondents were trained regarding the application of pesticides. The most prominent side effect of pesticides among respondents was tiredness (66.4\%).

Conclusion: Based on our findings, program is needed for training of pistachio farmers regarding methods of using pesticides and also how to use personal protection. It is also important to train respondents' families to prevent side effects of pesticides among their children.
\end{abstract}

Keywords: Pesticide, Pistachio farmers, Rafsanjan, Rural

\section{Introduction}

Living creatures' (human, animal, and plants) life is extensively at risk of exposure to chemical agents' (1). These hazardous materials varied from metallic elements and inorganic substances to heavy organic compounds. More recent attention has been paied to the biochemical, pathological and psychological aspects of these molecules by toxicologists (2).

Dangerous toxic agents consists of a wide range of materials including some sort of drugs, nutritional additives, insecticides, pesticides, industrial chemicals, air polluting agents and natural toxic substances (1-3). Of these, the most frequent poisoning reagents are detergents, pain killers, cosmetics, plants, anti cold and cough medications and hydrocarbons (4-6).

Different ways of exposure have been identified to date which are categorized as follow: a) eating $(70 \%)$, skin exposure $(7 \%)$, eye exposure $(6 \%)$, inhalation (5\%), animal biting, insects sting (3\%), and inflectional approach (3\%). Complications of a chemical reagent depends on

\footnotetext{
Corresponding author: Reza Vazirinejad, Social Determinants of Health Research Centre, Rafsanjan University of Medical Sciences, Rafsanjan, Iran. E-mail: rvazirinejad@yahoo.co.uk
} 
the concentrations and type of the reagents, period of exposure, and the presence of other materials in the environment. An annual number of 300,000 death is estimated due to the exposure to toxic substances worldwide, which mostly occur in developing countries. It is also expected that about five millions of people are at risk of exposure to toxic substances in USA, yearly (3).

In addition to the acute poisoning that causes thousands of death each year, lots of people are also suffering from several type of either psychological or non-psychological diseases due to exposing these substances (5). Working with pesticides is a seasonal career in Iran, specifically in Kerman province. These workers have more chance of exposure to these chemicals than normal population. Therefore, this study was designed to examine the health problems associated with exposing to pesticides and insecticides in workers of pistachio farms.

\section{Material and Methods}

This descriptive study was performed on workers whose job was working with pesticides in pistachio farms in rural area of Rafsanjan during 2010. A questionnaire was designed based on the aim and objectives of the study for the purpose of data collection.

The questionnaire contains two sections, the first section includes demographic information e.g. age, marital status, number of children, duration of working in pistachio farms, daily working hours, history of training in the field of working with toxic reagents (including insecticides and pesticide), using safety equipment during work with toxic reagent (e.g. mask, helmet, etc ...) and nutritional status during working days.

The second section of the questionnaire focused on more specific health status of farmers and consists of some other inquiries such as having a kid with an specific disease, history of referring to the hospital for the reason of exposure to poisonic reagents or any other reason. In this questionnaire safety equipment were categorised in six groups including mask, helmet, gloves, overall, boot and glasses. The commercial brands of insecticides which were applied by the farmers were also classified as Amitrase (Mitale), Endosulphan, Etium, Zolan, Fozolum, Paration, Ambush and Fonvalevite. Data about apetite, using liquids and fruits, farmers children disorders (history of dermatitis, sensitivity), leukemia, having atopic allergy, digestive disorders and diabetes were also collected by questionnaire.

All of the complications and symptoms that could occur following exposure to the pesticide in the farmers were also categorized in 19 categories and were asked as follow: 1headache 2- tiredness 3-insomnia 4- irritability 5- acedias 6- depression 7- adenauseam 8destruction 9- decentralization 10- anorexia 11heavy sweating 12-sight problems in night 13strabismus 14- tremor in hands 15- tachycardia 16- weakness 17 - body swelling 18 - provocation and 19- rubber faction.

A sample of 115 farmers were randomly selected from the list of all farmers in rural area of Rafsanjan. All of farmers were visited and interviewed in their workplaces by the research team. This part of data collection process was performed by getting help from the rural health centers.

Farmers with farming experience less than two years were excluded and replaced with others. Further, those farmers who had been recognized as having a disease un-related to their job were also excluded. Farmers who were between 18 to 100 years old and were working in farms as their permanent job were invited. Collected data were statistically analyzed using SPSS version 13. The parametric (T-test, Z-test, ANOVA) and non-parametric (Mann-withney, Chi-square) methods were applied where appropriate. 


\section{Results}

Respondents in this study were 115 randomly recruited male subjects with an average age of $44.5 \pm 14.5$ years. The minimum and maximum and age were 18 and 85 years, respectively. Our results indicated that $104(89.7 \%)$ cases were married and 11(10.3\%) were single. Less than $2 \%(\mathrm{n}=2,18 \%)$ of subjects were suffering from infertility. The mean duration of working was $20 \pm 12.6$ years and the mean working hours per day was $6.6 \pm 2.2$. The average of child number in each family of farmers was 5.2 \pm 2.6 (table 1).

Table-1: Some characteristic of farmers who accepted to participate

\begin{tabular}{ccccc}
\hline Characterizes & No & Mean \pm SD & Min & Max \\
\hline Age(years) & 115 & $44.5 \pm 14.5$ & 18 & 85 \\
$\begin{array}{c}\text { Occupation duration } \\
\quad \text { (year) }\end{array}$ & 115 & $20 \pm 12.6$ & 2 & 65 \\
$\begin{array}{c}\text { Daily working time } \\
\quad \text { (hour) }\end{array}$ & 115 & $6.6 \pm 2.5$ & 1 & 20 \\
$\begin{array}{c}\text { No. of children } \\
\text { o. }\end{array}$ & 102 & $5.2 \pm 2.6$ & 1 & 15 \\
\hline
\end{tabular}

Only $17(14.7 \%)$ of farmers received training for their job while the rest 98 (84.5\%) did not participate in training courses. Our results indicated that 44(37.9\%), 35(30.2\%), and 20 $(17.3 \%)$ of farmers used boot, mask, and helmet (and gloves) during the work, respectively.

Table -2: Some occupational characters of farmers in our study

\begin{tabular}{lcc}
\hline $\begin{array}{c}\text { Type of illness among farmers } \\
\text { children }\end{array}$ & No & \% \\
\hline - Mental disorder & 6 & 5.2 \\
- Pulmonary disorder & 7 & 6 \\
- Digestive disorder & 2 & 1.7 \\
- Skin disorder & 4 & 3.4 \\
- Thyroid disorder & 4 & 3.4 \\
- Leukemia & 1 & 0.9 \\
- Others & 4 & 3.4 \\
- Not changed & 51 & 44 \\
- Using milk products & 36 & 31 \\
- Liquids & 24 & 20.7 \\
- Fruits & 7 & 6 \\
- Appetite & 2 & 1.7 \\
- Amitraze & 103 & 88.8 \\
- Endosulphan & 95 & 82.6 \\
- Etium & 24 & 20.8 \\
- Zolam & 23 & 20 \\
- Fozolam & 7 & 6 \\
- Ambush & 10 & 8.6 \\
- Parathion & 2 & 1.7 \\
- Fonwalerite & 3 & 2.6 \\
- Pulmonary & 12 & 10.3 \\
- Joint & 12 & 10.3 \\
- Ocular & 7 & 6 \\
- Hematologic & 6 & 5.2 \\
- CNS & 5 & 4.3 \\
- Digestive & 2 & 1.7 \\
\hline
\end{tabular}


Only 4(3.7\%) subjects declared that they were glasses at work. None of farmers declared that they have especial nutritional schedule when they were spraying pesticides and only $6 \%$ reported eating fruits rich meals while working. Amitrase $(88.8 \%)$ and parathion $(1.7 \%)$ were applied more frequently. In compare to other pesticides, respectively. The most predominant disorder among farmers' children was mental retardation $(5.2 \%)$. Among farmers' children, $2.9 \%$ were born with congenital myelomeningocell. The most frequent reason for farmers referal to hospital was pulmonary and joint related disorders (1.7\%) (table 2). Digestive diseases were also the most ferquent cause for hospitalization (table-3).

Table-3: The distribution of farmers based on the sign and symptoms following working with pesticide and their hospitalization status

\begin{tabular}{lcc}
\hline \multicolumn{1}{c}{ Symptoms } & No & \% \\
\hline - Tiredness & 77 & 66.9 \\
- Headache & 65 & 56.5 \\
- Weakness in arms and feet & 45 & 39.1 \\
- Extensive sweating & 35 & 30.4 \\
- Tachycardia & 31 & 26.9 \\
- Dazing & 19 & 16.5 \\
- Appetites & 18 & 15.6 \\
- Vomiting & 16 & 13.9 \\
- Insomnia & 16 & 13.9 \\
- Depression & 15 & 13 \\
- Shake hands & 13 & 11.3 \\
- Red Eye & 13 & 11.3 \\
- Night blindness & 10 & 8.6 \\
- Anger face and body & 10 & 8.6 \\
- Distraction & 10 & 8.6 \\
- Irritability & 6 & 5.2 \\
- Strabismus & 4 & 3.4 \\
- Swelling of the face and organs & 4 & 3.4 \\
- Concentration disorders & 3 & 2.6 \\
Hospitalization Statue & & \\
- Surgery & 5 & 4.3 \\
- Cardiac disease & 4 & 3.4 \\
- Pulmonary disease & 4 & 3.4 \\
- Digestive disease & 6 & 5.2 \\
\hline
\end{tabular}

As table 5' shows, our results indicated that the most associated symptoms which farmers encounter following exposure to pesticides are categorized as: tiredness 77 (66.9\%), headache $65(56.5 \%)$, feeling weakness in arms and feet 45 (39.8\%), heavy sweating 35 (30.4\%), tachycardia 31 (26.9\%) and depression 15(13\%). Our findings also indicated that $4(3.6 \%)$ subjects had strabismus and 4 (3.6\%) suffered from organ swelling (table-3). There was no significant relation between the training status of farmers, regarding the methods of using safety equipment, and related complications (table-4). Five out of seven farmers informed our research group that had headache after using fozolum, 6 of them had tiredness and 4 had tachycardia. 
Table-4: The distribution of trained farmers based on the type of Personal Protective Equipment.

\begin{tabular}{cccc}
\hline \multirow{2}{*}{ Equipment } & Applied a PPE & Not applied a PPE & Total \\
\cline { 2 - 4 } & No (Percent) & No (Percent) & No (Percent) \\
\hline Mask & $4(23.5 \%)$ & $13(76.5 \%)$ & $17(100 \%)$ \\
Helmet & $3(17.6 \%)$ & $14(82.4 \%)$ & $17(100 \%)$ \\
Gloves & $5(29.4 \%)$ & $12(70.6 \%)$ & $17(100 \%)$ \\
Overall & $10(58.8 \%)$ & $7(41.2 \%)$ & $17(100 \%)$ \\
Boots & $6(35.3 \%)$ & $11(64.7 \%)$ & $17(100 \%)$ \\
Glasses & $1(5.9 \%)$ & $16(94.1 \%)$ & $17(100 \%)$ \\
\hline
\end{tabular}

\section{Discussion}

Pesticides are being widely applied for controlling insect and pests in agriculture. They are useful for palnts, in one side while hazardous for animal life on the otherside. Rafsanjan is globally recognized as the area for growing pistachio and having massive pistachio farms. To achieve a better yield of quantitative as well as qualitative pistachio' protection of this crops against insect is of the paramount importance. Thus, pesticides are widely applied for the above mentioned reasons. Therefore, in this study we enrolled 115 farmers during the beginning of the summer of 2010. Our study was limited to the farmers in pistachio farms and most often commercial pesticides including used Amitraz (88.8\%), Endosulohan (82.9\%), Etium (20.7\%), Zolam (19.3\%), Ambush (8.6\%), Fuzolam (6\%), Fonvalverite $(2.6 \%)$ and Paration (1.7\%).

The results of a 5 years follow up study by Kamel and colleagues (2005) also shows that the most reagents which are being used are organophasphorates and organoclorines $(7,9)$. Our study indicated that tiredness, headache, feeling weakness in feet and hands, extensive sweating, sleeping disorders and insomnia, adnauseam, visional complications and distraction were frequent symptoms of toxicity with pesticides. In a similar study, Frey and colleagues (18782 subjects) reported that headache, tiredness, insomnia, depression, distraction, apetite, extensive sweating, tachycardia, feeling of weakness in legs and arms, visional difficulties, caution difficulties were also the most frequent complications in this type of workers $(7,9,10,11)$. We showed a prevalence of $6 \%$ (7 individuals) of pulmonary disease, $5.2 \%$ (6 individuals) mental disorders, skin and thyroid disorders $3.4 \%$ (4 in each group) and only $0.9 \%$ (one person) of leukemia in the farmers children. Wayne (2003) reported that congenital disorders are more prevalent in farmers children compared to normal children and also the occurrence of non-Hodgkin's lymphoma is related to the pesticides exposure (12).

In concert with our findings Garry's and colleagues reported that among 210723 live birth in Minnesota the rate of congenital disorders in the farmers' children is higher than that among other groups (2003) and most often among children born a month after spring. Taken together it my indicate that the disorders were established at the first trimester of embryonic period which could be affected by pesticides $(13,15)$.

We also observed a lesser extending in fertility prevalence $(18 \%)$ in our study population. This is in contrast to the reported prevalence by Charbel and worker who showed a $28 \%$ prevalence. This discrepancy could probably be due to the sample size of the study, because we only studied 115 cases whilst Charbel studied population was 80 workers (18). Our findings showed that only 4 (3.4\%) farmers had a history of poisoning during spraying pesticides. In contrast to this study, Ghazi Khansari and colleagues did not find any cases of poisoning during the work period in farmers (17). In our 
study the most prevalent diseases were ocular diseases $6 \% \quad(7$ subjects $)$, hematological disorders 5.2\% (6 subjects) and CNS disorders $4.3 \%$ (5 subjects). In agreement with this study Michael indicated that insecticides could affect nervous, immune and genital systems (18). Following up study shown that the first trimester of the respected mothers pregnancy was coincide with the time of massive insecticide application in pistachio farms.

Overall, according to the findings of our investigation it could be suggested that:

1. Due to the deleterious effects of the pesticides on the environment, human, wild and domestic animals more research programs need to be financially fund by Iranian government to find out some favorable methods to control thes unwanted effects.

2. To train and educate farmers and their families regarding how to use these hazardous materials, side effects of these reagents on their health.

3. To establish training programs and instruction to farmers regarding methods of application of personal protection equipments during work with pesticides and other poisonous reagents.

4. To dispatch personal protection equipments with farmers.

5. Establishment of an information network to collect more information about side effects of pesticides on farmers and environment health.

\section{Conclusion}

Finally more studies are needed to investigate the deleterious effects of pesticides and other compounds on the other parts of the country with a larger sample size. Further, all per mental based examinations are also useful to find out how, these poisoning materials effect body systems (e.g. digestive, pulmonary ...) and specific organs like liver, kidney in farmers. Due to ethics limitations, to achieve better understanding on the effects of pesticides, it is essential to assess, how exposure to these materials specially (one by one for each pesticides) influence the organs functions among animal labs. More over, due to the importance of this issue it is of paramount importance to look at the side effects of other pesticides which are widely used for other crops rather than pistachio in other regions of the country.

\section{Acknowledgments}

We warmly appreciate all of farmers who participated in this research programme. This study was financially supported by a grant from Rafsanjan University of Medical Sciences.

\section{Conflict of interest}

None of the authors of this article declared conflict of interest.

\section{References}

1. Tamberl J, Salar-Amoli J (2000). Introduction to Toxicology. Jahad -daneshgahi: Tehran. Chapter 1. [Farsi]

2. Klassen CD, Casarett DS (1996). Toxicology the Basic science of poisons, 5th ed. NewYork: McGraw-Hill. P 23-25.

3. WHO, Hazardous chemicals in human and environmental Health 2000.

4. Ward MH, Nuckols JR, Weigel SJ, Maxwell SK, Cantor KP, Miller RS. Identifying populations potentially posed to Agricultoral pesticides, Environ Health perspect 2000; 108(1):5-12.

5. Eamaili A, Vazirinejad R (2006). Safety principles to work with chemicals. ${ }^{\text {th }}$ ed. Razban: Tehran. Chapter 3. [Farsi]

6. Butkin D, Koler E (2003). Vahabzade Ab. Environmental Recognition. Jahad -daneshgahi: Mashhad. Chapter 1. [Farsi]

7. Kamel F, Engel LS, Gladen BC, Hoppin JA, Alavanja MC, Sandler DP. Neurologic symptoms in Licensed private pesticide Applicators in the 
Agricultural Health study. Environ Health perspect 2005; 113(7):877-82.

8. Starks SE, Hoppin JA, Kamel F, Lynch CF, Jones MP, Alavanja MC et.al. Peripheral Nervous System Function and Organophosphate Pesticide Use among Licensed Pesticide Applicators in the Agricultural Health Study. Environ Health Perspect 2012; 120(4):515-20.

9. Starks SE, Gerr F, Kamel F, Lynch CF, Alavanja MC, Sandler DP et.al. High pesticide exposure events and central nervous system function among pesticide applicators in the Agricultural Health Study. Int Arch Occup Environ Health 2012; 85(5):505-15.

10. Kamel F, Engel LS, Gladen BC, Hoppin JA, Alavanja MCR, Sandler DP. Neurologic symptoms in licensed private pesticide applicators in the Agricultural Health Study. Environ Health Perspect 2005; 113(7):877-82.

11. Stallones L, Beseler C. Pesticide Poisoning and Depressive symptoms among Farm Residents, Ann Epidemol 2002; 12(6):389-94.

12. Sinclair W, Pressinger R. Birth Defects Higher in Babies Born to Families Living near Farming Areas using Pesticides. Environmental Health perspectives 2003; 111(9):1259-64.
13. Garry VF, Schreinemachers D, Harkins ME, Griffith J. Pesticide appliers, biocides, and birth defects in rural Minnesota. Environ Health Perspect. 1996; 104(4):394-9.

14. Garry VF, Harkins ME, Erickson LL, LongSimpson LK, Holland SE, Burroughs BL. Birth defects, season of conception, and sex of children born to pesticide applicators living in the Red River Valley of Minnesota, USA. Environ Health Perspect 2002; 110(3):441-9.

15. Garry VF, Holland SE, Erickson LL, Burroughs BL. Male reproductive hormones and thyroid function in pesticide applicators in the Red River Valley of Minnesota. J Toxicol Environ Health A 2003; 66(11):965-86.

16. Massaad C, Entezami F, Massade L, Benahmed $\mathrm{M}$, Olivennes $\mathrm{F}$, Barouki $\mathrm{R}$ et.al. How can chemical compounds alter human fertility? Eur J Obstet Gynecol Reprod Biol 2002; 100(2):12737.

17. Ghazi - khansari M, Oreizi S. A prospective study of fatal outcomes of poisoning in Tehran. Vet Hum Toxicol 1995; 37(5):449-52.

18. O'Malley M. Clinical evaluation of pesticide exposure and poisoning. Lancet 1997; 349(9059): 1161-6. 